\title{
Cassini- and Catalan-like formulas for polygonal numbers and simplex numbers
}

\author{
Thomas Jeffery
}

\begin{abstract}
This study was conducted under the supervision of Professor Allan Willms, Department of Mathematics and Statistics, College of Physical and Engineering Science, University of Guelph
\end{abstract}

\begin{abstract}
Cassini's formula and Catalan's formula are two results from the theory of Fibonacci numbers. This article derives results similar to these, however instead of applying to Fibonacci numbers, they are applied to polygonal numbers and simplex numbers. Triangular numbers are considered first. We then generalize to polygonal and simplex numbers. For polygonal numbers the properties of determinants are used to simplify calculations. For simplex numbers Pascal's Theorem is used.
\end{abstract}

\section{INTRODUCTION}

The Fibonacci sequence is defined by the following recurrence relation:

\section{Definition 1:}

$F_{n}=F_{n-1}+F_{n-2} \quad, F_{1}=F_{2}=1$

The first few Fibonacci numbers are: $1,1,2,3,5,8,13,21, \ldots$

The following proposition follows from Definition 1.

\section{Proposition 1:}

$F_{n}=\frac{\alpha^{n}-\beta^{n}}{\sqrt{5}}$, where $\alpha=\frac{1+\sqrt{5}}{2}$ and $\beta=\frac{1-\sqrt{5}}{2}$

Definition 1 is usually referred to as the Fibonacci recurrence relation. Proposition 1 is known as Binet's Formula. Cassini's formula, and to a lesser extent Catalan's formula, are two of the most fundamental results in the theory of Fibonacci numbers. These two formulas are as follows:

\section{Cassini's Formula:}

$F_{n-1} F_{n+1}-F_{n}{ }^{2}=(-1)^{n}$, for every positive integer $\mathrm{n}$

\section{Catalan's Formula:}

$F_{n-k} F_{n+k}-F_{n}^{2}=F_{k}^{2}(-1)^{n+k+1}$, for all positive integers $\mathrm{n}$ and $\mathrm{k}$ where $k<n$

For proofs of Binet, Cassini, and Catalan formulas see [1]. This article addresses the following two problems:

\section{Problem 1:}

Given the sequence: $\left\{x_{n}\right\}$, we would like to find a simple characterization of the sequence $\left\{y_{n}\right\}$, such that:

$x_{n-1} x_{n+1}-x_{n}{ }^{2}=y_{n}$

Throughout this article the previous equation shall be called Cassini's formula for the sequence $\left\{x_{n}\right\}$. 


\section{Problem 2:}

Given the sequence: $\left\{x_{n}\right\}$, we would like to find a simple characterization of the sequence $\left\{y_{n, k}\right\}$, such that:

$x_{n-k} x_{n+k}-x_{n}{ }^{2}=y_{n, k}$

Throughout this article the previous equation shall be called Catalan's formula for the sequence $\left\{x_{n}\right\}$.

Clearly, Cassini's formula is a special case of Catalan's formula. Thus, if we can solve Problem 2, then the solution to

Problem 1 will follow as the special case where $\mathrm{k}=1$. The focus of this article is on solving these two problems for two special classes of sequences. These are the sequence of polygonal numbers and the sequence of simplex numbers. Section 2 of this article solves Problems 1 and 2 for the sequence of triangular numbers. Triangular numbers are a good place to start because, as the reader will soon see, they are both polygonal numbers and simplex numbers. Section 3 of this article solves Problem 2 for the sequence of polygonal numbers. The solution to Problem 1 for polygonal numbers then follows as a corollary. Finally, Section 4 of this article solves Problem 1 for the sequence of simplex numbers.

\section{Triangular Numbers}

The sequence of triangular numbers is defined by the following recurrence relation:

\section{Definition 2}

$t_{0}=1, t_{n}=t_{n-1}+n, n>0$

Triangular numbers have the property that if $t_{n}$ is a triangular number then $t_{n}$ objects can be arranged to form a triangle. The first four triangular numbers are: $1,3,6$, and 10 . These four numbers can be arranged into triangles as follows: 
It can be shown that the following Proposition follows from Definition 2.

\section{Proposition 2}

$t_{n}=\sum_{i=1}^{n} i=\frac{n(n+1)}{2}$

We now derive a Cassini like formula for the sequence of triangular numbers using Proposition 2:

$$
\begin{aligned}
t_{n-1} t_{n+1}-t_{n}^{2}= & \frac{(n-1)(n)}{2} \frac{(n+1)(n+2)}{2}-\left(\frac{n(n+1)}{2}\right)^{2} \\
& =\frac{n(n+1)}{4}((n-1)(n+2)-n(n+1)) \\
& =\frac{n(n+1)}{4}\left(n^{2}+n-2-n^{2}-n\right) \\
& =\frac{n(n+1)}{4}(-2) \\
& =\frac{-n(n+1)}{2} \\
& =-t_{n}
\end{aligned}
$$

Thus we have the following Theorem.

\section{Theorem 1: (Cassini's Formula for Triangular Numbers)}

$t_{n-1} t_{n+1}-t_{n}{ }^{2}=-t_{n}$, for every positive integer $n>1$.

This theorem appears in [2]. Also take note, that every major result derived in this article, is in some way or another, a generalization of Theorem 1.

We now derive a generalization of Theorem 1. Once again we shall use Proposition 2.

$$
\begin{aligned}
& t_{n-k} t_{n+k}-t_{n}{ }^{2}=\frac{(n-k)(n-k+1)}{2} \frac{(n+k)(n+k+1)}{2}-\left(\frac{n(n+1)}{2}\right)^{2} \\
& =\frac{1}{4}\left((n-k)(n+k)((n+1)-k)((n+1)+k)-n^{2}(n+1)^{2}\right) \\
& =\frac{1}{4}\left(\left(n^{2}-k^{2}\right)\left((n+1)^{2}-k^{2}\right)-n^{2}(n+1)^{2}\right) \\
& =\frac{1}{4}\left(\left(n^{2}-k^{2}\right)(n+1)^{2}+-k^{2}\left(n^{2}-k^{2}\right)-n^{2}(n+1)^{2}\right) \\
& =\frac{1}{4}\left((n+1)^{2}\left(-k^{2}\right)-k^{2}\left(n^{2}-k^{2}\right)\right) \\
& =\frac{k^{2}}{4}\left((-1)\left(n^{2}+2 n+1\right)-\left(n^{2}-k^{2}\right)\right) \\
& =\frac{k^{2}}{4}\left(-2 n^{2}-2 n+k^{2}-1\right) \\
& =\frac{k^{2}}{4}\left(k^{2}-1\right)+\frac{k^{2}}{4}\left(-2 n^{2}-2 n\right) \\
& =\frac{k^{2}}{4}(k+1)(k-1)+\frac{k^{2}}{4}(-2 n)(n+1) \\
& =\frac{k(k+1)}{2} \frac{(k-1) k}{2}-k^{2} \frac{n(n+1)}{2} \\
& =t_{k} t_{k-1}-k^{2} t_{n}
\end{aligned}
$$

Thus, we have proved:

\section{Theorem 2: (Catalan's Formula for Triangular Numbers)}

$t_{n-k} t_{n+k}-t_{n}{ }^{2}=t_{k} t_{k-1}-k^{2} t_{n}$, where $\mathrm{n}$ and $\mathrm{k}$ are positive integers and $k<n$

We now present an alternative proof of Theorem 2. This proof involves the following lemmas. Lemma 1 is the definition of the determinant of a two by two matrix. Lemmas 2 and 3 are properties of determinants of a two by two matrix. These properties can be generalized to a square matrix of any size see [3], however for the purpose of this article, properties of two by two matrices will suffice. The theory of determinants simplifies the alternate proof of theorem 2 although it is not entirely necessary. Lemma 4, which appears in [2], states that the sum of two consecutive triangular numbers is a square number. Lemmas 5 and 6, which also appear in [2], follow from Proposition 2 of triangular numbers as a sum.

\section{Lemma 1}

$\left|\begin{array}{ll}a_{11} & a_{12} \\ a_{21} & a_{22}\end{array}\right|=a_{11} a_{22}-a_{12} a_{21}$ 


\section{Lemma 2}

$\left|\begin{array}{ll}a_{11} & a_{12} \\ a_{21} & a_{22}\end{array}\right|=\left|\begin{array}{cc}a_{11} & a_{12} \\ a_{21}+c a_{11} & a_{22}+c a_{12}\end{array}\right|$, for all $c \in \mathbb{R}$

\section{Lemma 3}

$\left|\begin{array}{ll}a_{11} & a_{12} \\ a_{21} & a_{22}\end{array}\right|=\left|\begin{array}{ll}a_{11}+c a_{12} & a_{12} \\ a_{21}+c a_{22} & a_{22}\end{array}\right|$, for all $c \in \mathbb{R}$

\section{Lemma 4}

$$
\begin{aligned}
& t_{k}+t_{k-1}=k^{2} \\
& \text { Proof } \\
& \begin{aligned}
t_{k}+t_{k-1}= & \frac{k(k+1)}{2}+\frac{(k-1) k}{2} \\
& =\frac{k}{2}((k+1)+(k-1)) \\
& =\frac{k}{2}(2 k) \\
& =k^{2}
\end{aligned}
\end{aligned}
$$

\section{Lemma 5}

$$
\begin{aligned}
& \begin{aligned}
t_{n-k} & =t_{n}-n k+t_{k-1} \\
\text { Proof } & \\
t_{n-k} & =\sum_{i=1}^{n-k} i, \text { by Proposition } 2 \\
& =\sum_{i=1}^{n} i-\sum_{i=n-k+1}^{n} i \\
& =t_{n}-((n-k+1)+(n-k+2)+\cdots+(n-1)+n) \\
& =t_{n}-\left(n k-\sum_{i=1}^{k-1} i\right), \text { since there are } k \text { terms in this series } \\
& =t_{n}-n k+t_{k-1}, \text { by Proposition } 2
\end{aligned}
\end{aligned}
$$

\section{Lemma 6}

$$
\begin{aligned}
& t_{n+k}=t_{n}+n k+t_{k} \\
& \text { Proof } \\
& \begin{aligned}
t_{n+k}= & \sum_{i=1}^{n+k} i, \text { by Definition } \\
& =\sum_{i=1}^{n} i+\sum_{i=n+1}^{n+k} i \\
& =t_{n}+((n+1)+(n+2)+\cdots+(n+k-1)+(n+k)) \\
& =t_{n}+n k+\sum_{i=1}^{k} i, \text { since there are } k \text { terms in this series } \\
& =t_{n}+n k+t_{k}
\end{aligned}
\end{aligned}
$$

We are now ready to give the alternate proof of Theorem 2. We begin by expressing the left side of the equation in terms of determinants. We then simplify the expression using the previous lemmas.

$$
\begin{aligned}
& t_{n-k} t_{n+k}-t_{n}{ }^{2}=\left|\begin{array}{cc}
t_{n+k} & t_{n} \\
t_{n} & t_{n-k}
\end{array}\right|, \text { by Lemma } 1 \\
&=\left|\begin{array}{cc}
t_{n}+n k+t_{k} & t_{n} \\
t_{n} & t_{n}-n k+t_{k-1}
\end{array}\right| \text {, by Lemmas } 5 \text { and } 6 \\
&=\left|\begin{array}{cc}
t_{n}+n k+t_{k} & t_{n} \\
-n k-t_{k} & -n k+t_{k-1}
\end{array}\right|, \text { by Lemma } 2 \text { with } c=-1 \\
&= \begin{array}{cc}
n k+t_{k} \\
-t_{k}-t_{k-1} & -n k+t_{k-1}
\end{array} \mid, \text { by Lemma } 3 \text { with } c=-1 \\
&=\left(n k+t_{k}\right)\left(-n k+t_{k-1}\right)-t_{n}\left(-t_{k}-t_{k-1}\right), \text { by Lemma } 1 \\
&=-n^{2} k^{2}-n k t_{k}+n k t_{k-1}+t_{k} t_{k-1}+k^{2} t_{n}, \text { by Proposition } 2 \\
&=-n^{2} k^{2}-n k\left(t_{k}-t_{k-1}\right)+t_{k} t_{k-1}+k^{2} t_{n} \\
&=-n^{2} k^{2}-n k k+t_{k} t_{k-1}+k^{2} t_{n}, \text { by lemma } 4 \\
&= t_{k} t_{k-1}+k^{2}\left(t_{n}-n^{2}-n\right) \\
&= t_{k} t_{k-1}+k^{2}\left(t_{n}-2 t_{n}\right), \text { by Proposition } 2 \\
&= t_{k} t_{k-1}-k^{2} t_{n} \\
& \text { QED }
\end{aligned}
$$




\section{Polygonal Numbers}

Polygonal numbers are defined by the following recurrence relation:

\section{Definition 3}

$p_{1}^{m}=1, p_{n}^{m}=p_{n-1}^{m}+(m-2) n-(m-3), \mathrm{m}$ and $\mathrm{n}$ are positive integers, $\mathrm{m}>1, \mathrm{n}>1$

Polygonal numbers have the property that if $p_{n}^{m}$ is the nth $\mathrm{m}$-gonal number then $p_{n}^{m}$ objects can be arranged to form a polygon with $m$ sides. For example $p_{4}^{5}=22$ and so 22 objects can be arranged to form a pentagon as follows:

The following propositions follow from Definition 3.

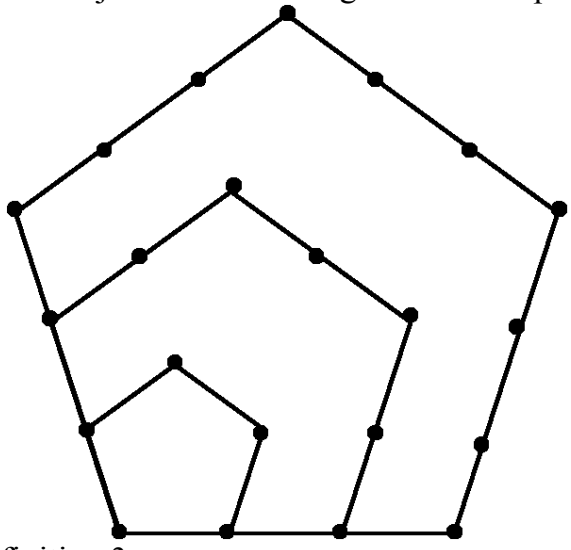

\section{Proposition 3}

$p_{n}^{m}=\sum_{i=1}^{n}((m-2) i-(m-3))=\frac{n}{2}((m-2) n+4-m)$

we let $m=3$ then Definition 3 is the same as Definition 2, while Proposition 3 is the same as Proposition 2. But Definition 2 and Proposition 2 involve triangular numbers. Thus, $p_{n}^{3}=t_{n}$, so the polygonal numbers are a generalization of the triangular numbers. It now follows that the Catalan like formula for the polygonal numbers, which we will derive shortly, is a generalization of Theorem 2. The proof of the general version of Theorem 2 is very similar to the second proof of Theorem 2. Once again determinants are used to simplify the calculations. First we require two lemmas, which are generalizations of Lemmas 5 and 6 .

\section{Lemma 7}

$$
\begin{aligned}
& p_{n-k}^{m}=p_{n}^{m}-(m-2) n k+(m-2) t_{k-1}+(m-3) k \\
& \text { Proof } \\
& \begin{aligned}
p_{n-k}^{m}=\sum_{i=1}^{n-k}((m-2) i-(m-3)) \\
\quad=\sum_{i=1}^{n}((m-2) i-(m-3))-\sum_{i=n-k+1}^{n}((m-2) i-(m-3)) \\
\quad=p_{n}^{m}-((m-2)((n-k+1)+\cdots+(n))-(m-3)(k)) \\
\quad=p_{n}^{m}-\left((m-2)\left(n k-t_{k-1}\right)-(m-3) k\right) \\
\quad=p_{n}^{m}-(m-2) n k+(m-2) t_{k-1}+(m-3) k
\end{aligned}
\end{aligned}
$$

\section{Lemma 8}

$$
\begin{aligned}
& \begin{array}{l}
p_{n+k}^{m}=p_{n}^{m}+ \\
\text { Proof }
\end{array} \\
& \begin{aligned}
p_{n+k}^{m}=\sum_{i=1}^{n+k} & \left((m-2) n k+(m-2) t_{k}-(m-3) k\right. \\
& =\sum_{i=1}^{n}((m-2) i-(m-3))+\sum_{i=n+1}^{n+k}((m-2) i-(m-3)) \\
& =p_{n}^{m}+(m-2)((n+1)+\cdots+(n+k))-(m-3)(k) \\
& =p_{n}^{m}+(m-2)\left(n k+t_{k}\right)-(m-3) k \\
& =p_{n}^{m}+(m-2) n k+(m-2) t_{k}-(m-3) k
\end{aligned}
\end{aligned}
$$

Using Lemmas 7 and 8, along the properties of determinants, we now derive Catalan's formula for polygonal numbers. $p_{n-k}^{m} p_{n+k}^{m}-\left(p_{n}^{m}\right)^{2}=\left|\begin{array}{cc}p_{n+k}^{m} & p_{n}^{m} \\ p_{n}^{m} & p_{n-k}^{m}\end{array}\right|$ 


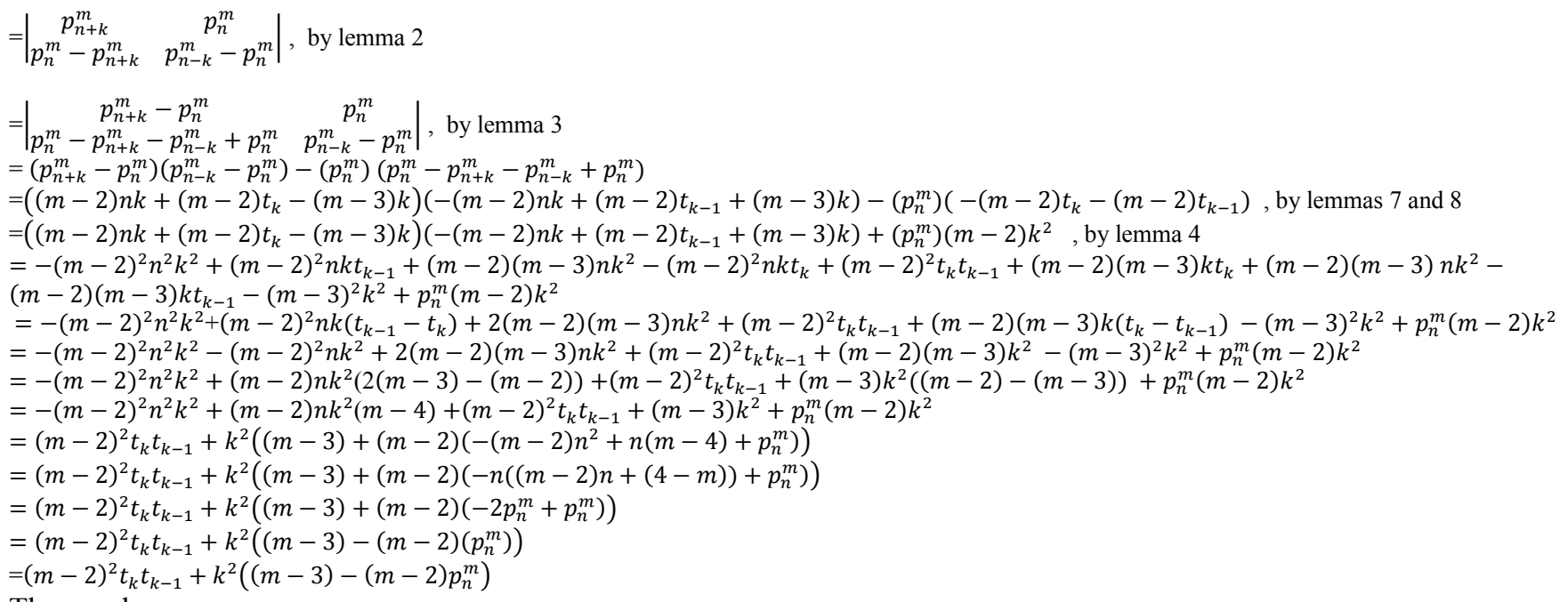

Thus we have

\section{Theorem 3 (Catalan's Formula for Polygonal Numbers)}

$p_{n-k}^{m} p_{n+k}^{m}-\left(p_{n}^{m}\right)^{2}=(m-2)^{2} t_{k} t_{k-1}+k^{2}\left((m-3)-(m-2) p_{n}^{m}\right)$,

where $n, k$, and $m$ are positive integers, $k<n, m>1$

\section{SimpleX NumberS}

Simplex numbers are defined by following recurrence relation:

\section{Definition 4}

$t_{1}^{m}=1, t_{n}^{m}=t_{n-1}^{m}+t_{n}^{m-1}, n>1, m>1$

An m-simplex is an m dimensional equivalent of a triangle. M-simplex numbers have the property that if $t_{n}^{m}$ is the nth $\mathrm{m}$ simplex number then $t_{n}^{m}$ objects can be arranged to form an m-simplex. M-simplex numbers also appear as the diagonal entries in Pascal's triangle. That is to say, that $t_{n}^{m}$ is the nth entry on the mth diagonal of Pascal's triangle.

Definition 4 can be used to prove the following two Propositions.

\section{Proposition 4}

$t_{n}^{m}=\sum_{i=1}^{n} t_{i}^{m-1}$

\section{Definition 5}

The factorial function denoted $n !$ read as "n factorial" is defined as follows:

$$
n !=n(n-1)(n-1) \ldots(3)(2)(1)
$$

Also we take $0 !=1$

\section{Definition 6}

The binomial coefficient denoted $\left(\begin{array}{l}n \\ r\end{array}\right)$ read as "n choose $\mathrm{r}$ " is defined as follows:

$\left(\begin{array}{l}n \\ r\end{array}\right)=\frac{n !}{r !(n-r) !}$

\section{Proposition 5}

$t_{n}^{m}=\left(\begin{array}{c}n+m-1 \\ m\end{array}\right)$

If we let $m=2$, definition 4 reduces to Definition 2 and Propositions 4 and 5 reduce to Proposition 2 since $t_{n}^{2}=\sum_{i=1}^{n} t_{i}^{1}$ and $t_{n}^{2}=\left(\begin{array}{c}n+1 \\ 2\end{array}\right)=\frac{n(n+1)}{2}$. Thus, triangular numbers are a special case of simplex numbers. That is, $t_{n}^{2}=t_{n}$.

We now derive a Cassini like formula for simplex numbers using the following Lemma, which is commonly known as Pascal's Theorem. 


\section{Lemma 9 (Pascal's Theorem)}

$\left(\begin{array}{l}n \\ r\end{array}\right)=\left(\begin{array}{c}n-1 \\ r\end{array}\right)+\left(\begin{array}{c}n-1 \\ r-1\end{array}\right)$

We omit the proof.

By Pascal's Theorem and Proposition 5 we have:

$$
\begin{aligned}
t_{n-1}^{m} t_{n+1}^{m}-\left(t_{n}^{m}\right)^{2}=\left(\begin{array}{c}
n-2+m \\
m
\end{array}\right)\left(\begin{array}{c}
n+m \\
m
\end{array}\right)-\left(\begin{array}{c}
n+m-1 \\
m
\end{array}\right)^{2} \\
=\left\{\left(\begin{array}{c}
n-1+m \\
m
\end{array}\right)-\left(\begin{array}{c}
n-2+m \\
m-1
\end{array}\right)\right\}\left\{\left(\begin{array}{c}
n+m-1 \\
m
\end{array}\right)+\left(\begin{array}{c}
n+m-1 \\
m-1
\end{array}\right)\right\}-\left(\begin{array}{c}
n+m-1 \\
m
\end{array}\right)^{2} \\
=\left(\begin{array}{c}
n-1+m \\
m
\end{array}\right)\left(\begin{array}{c}
n+m-1 \\
m-1
\end{array}\right)-\left(\begin{array}{c}
n-2+m \\
m-1
\end{array}\right)\left(\begin{array}{c}
n+m-1 \\
m
\end{array}\right)-\left(\begin{array}{c}
n-2+m \\
m-1
\end{array}\right)\left(\begin{array}{c}
n+m-1 \\
m-1
\end{array}\right) \\
\left.=\left(\begin{array}{c}
n-1+m \\
m
\end{array}\right)\left(\begin{array}{c}
n+m-1 \\
m-1
\end{array}\right)-\left(\begin{array}{c}
n-2+m \\
m-1
\end{array}\right)\left(\begin{array}{c}
n+m-1 \\
m
\end{array}\right)+\left(\begin{array}{c}
n+m-1 \\
m-1
\end{array}\right)\right) \\
=\left(\begin{array}{c}
n-1+m \\
m
\end{array}\right)\left(\begin{array}{c}
n+m-1 \\
m-1
\end{array}\right)-\left(\begin{array}{c}
n-2+m \\
m-1
\end{array}\right)\left(\begin{array}{c}
n+m \\
m
\end{array}\right) \text {, by Pascal's Theorem } \\
=t_{n}^{m} t_{n+1}^{m-1}-t_{n}^{m-1} t_{n+1}^{m}, \text { by Proposition } 5
\end{aligned}
$$

We now have the following theorem.

\section{Theorem 4 (Cassini's Formula for Simplex Numbers)}

$$
t_{n-1}^{m} t_{n+1}^{m}-\left(t_{n}^{m}\right)^{2}=t_{n}^{m} t_{n+1}^{m-1}-t_{n}^{m-1} t_{n+1}^{m}
$$

\section{CONCLUSION}

In this paper we have derived Cassini and Catalan like formulas for triangular numbers. We then generalized by using the theory of determinants to derive similar formulas for polygonal numbers. Clearly, Cassini and Catalan like formulas for triangular numbers are a special case of Cassini and Catalan like formulas for polygonal numbers. We then generalized in another direction by using Pascal's Theorem to derive a Cassini like formula for simplex numbers.

\section{REFERENCES}

1. T. Koshy, Fibonacci and Lucas Numbers with Applications, Wiley, New York, 2001.

2. T Trotter, Jr., 1973, "Some Identities for Triangular Numbers," Journal of Recreational Mathematics, 6:2, 127-135.

3. H. Anton, Elementary Linear Algebra, $4^{\text {th }}$ ed., Wiley, 1984. 